\section{Subglottic Stenosis Is a Form of Limited Wegener's Granulomatosis}

\section{To the Editor:}

In a recent report in The Journal, Pagnoux, et al ${ }^{1}$ performed a retrospective analysis of patients with localized granulomatosis with polyangiitis (Wegener's, GPA) from the French Vasculitis Study Group database, which accounts for almost 500 patients collected from 1982 to 2003. The aim of the study was to estimate the frequency and describe the characteristics of patients with strictly localized disease that did not progress to a generalized form. In their analysis, Pagnoux, et al searched for patients with disease limited to only one organ and with followup of more than a year, to ensure that there was no progression to a systemic form of disease. Compared to previous reports, they found a relatively lower prevalence of the limited form of GPA: $3.2 \%$ vs $15 \%-30 \% \%^{2,3}$. Interestingly, in their group of 16 patients only 3 forms of limited disease were found, including nose and sinus involvement, orbital pseudotumors, and lung nodules. Of interest, no patient with subglottic stenosis was included, considering that this form by itself is a well recognized form of localized $\mathrm{GPA}^{4}$.

We describe a patient with longterm localized subglottic stenosis that did not evolve into a systemic form on followup of several years.

A 73-year-old woman referred to our clinic in April 2009 for evaluation of possible GPA presented with a 10-year history of shortness of breath, which had worsened over the past 2 years. Tracheal biopsy showed ulceration with acutely and chronically inflamed granulation tissue at the base of the ulcer, with focal dense fibrous tissue. Repeated antineutrophil cytoplasmic antibody (ANCA) tests were negative. In January 2009, she experienced a life-threatening episode of respiratory distress that required emergency tracheotomy diagnosed as subglottic stenosis. Extensive diagnostic investigation ruled out underlying malignancy, infections, and amyloid disease. Over the next 18 months, her clinical course was characterized by severe episodes of shortness of breath and hemoptysis, only partially responsive to prednisone therapy up to $60 \mathrm{mg}$ daily, methotrexate (MTX) up to $25 \mathrm{mg}$ weekly, and trimethoprim/sulfamethoxazol (T/S DS) 3 times per week. In July 2010, she was hospitalized for a severe episode of shortness of breath and hemoptysis, and ear-nose-throat (ENT) evaluation showed dramatically narrowed subglottic region with essentially no airway. After 3 months of unsuccessful clinical response with high-dose prednisone and MTX, a decision was made to use rituximab $500 \mathrm{mg} 2$ doses 2 weeks apart. Following the first dose her symptoms had mildly improved and ENT evaluation showed significant improvement, with 50\% limitation of the airway space.

At 3-month followup, there was no shortness of breath, no hemoptysis episodes, prednisone was down to $5 \mathrm{mg}$ daily, MTX was withdrawn, T/S DS continued; more significantly ENT evaluation revealed subglottic region markedly improved with only $5 \%$ limitation of the airway, and the patient was able to breathe easily with the tracheotomy plugged.

Our case clearly illustrates that subglottic stenosis should be considered a localized form of GPA, and conforms with the European League Against Rheumatism/European Vasculitis Study Group definition that localized disease is expected to be ANCA-negative ${ }^{5}$. Holle, et $a l^{6}$ from Germany also analyzed their cohort of suspected GPA of 1024 patients from 1989 to 2009 , and found that 50 patients $(5 \%)$ had localized disease restricted to the upper and/lower respiratory tract and orbital masses, and subglottic stenosis was present in $15 \%$ of their cohort. As in our patient, whose clinical course was characterized by refractoriness to conventional therapy and a relapsing course and required surgical intervention, a significant proportion of patients with limited disease, particularly those with subglottic stenosis in the Holle, et al series, also required cytotoxic therapy, exhibited relapse, and needed surgery ${ }^{6}$.

Rituximab was shown to be highly effective in our patient, and confirmed similar reports of its use in patients with subglottic stenosis ${ }^{7}$. However, we concur with Pagnoux, et al and Holle, et al that further prospective studies are needed with a larger number of patients and longer followup to better characterize this subset of patients with GPA and define their therapeutic management.

IGNACIO GARCIA-VALLADARES, MD; LUIS R. ESPINOZA, MD, Section of Rheumatology, LSU Health Sciences Center, New Orleans, Louisiana 70115, USA. Address correspondence to Dr. Espinoza. E-mail: lespin1@Isuhsc.edu

\section{REFERENCES}

1. Pagnoux C, Stubbe M, Lifermann F, Decaux O, Pavic M, Bérezné $\mathrm{A}$, et al. Wegener's granulomatosis strictly and persistently localized to one organ is rare: assessment of 16 patients from the French Vasculitis Study Group database. J Rheumatol 2011; 38:475-8.

2. Matteson EL, Gold KN, Bloch DA, Hunder GG. Long-term survival of patients with Wegener's granulomatosis from the American College of Rheumatology Wegener's granulomatosis classification criteria cohort. Am J Med 1996;101:129-34.

3. Reinhold-Keller E, Beuge N, Latza U, de Groot K, Rudert H, Nölle $\mathrm{B}$, et al. An interdisciplinary approach to the care of patients with Wegener's granulomatosis: long-term outcome in 155 patients. Arthritis Rheum 2000;43:1021-32.

4. Rasmussen N, Jayne DR, Abramowicz D, Bacon P, Cohen-Tervaert JW, Guillevin L, et al. European therapeutic trials in ANCA-associated systemic vasculitis: disease scoring, consensus regimens and proposed clinical trials. Clin Exp Immunol 1995;101 Suppl 1:29-43.

5. Helmich B, Flossmann O, Gross WL, Bacon P, Cohen-Tervaert JW, Guillevin L, et al. EULAR recommendations for conducting clinical studies and/or clinical trials in systemic vasculitis: focus on anti-neutrophil cytoplasm antibody-associated vasculitis. Ann Rheum Dis 2007;66:605-17.

6. Holle JU, Gross WL, Holl-Ulrich K, Ambrosch P, Noelle B, Both $\mathrm{M}$, et al. Prospective long-term follow-up of patients with localized Wegener's granulomatosis: does it occur as persistent disease stage? Ann Rheum Dis 2010;69:1934-9.

7. Solans Laque R. Clinical characteristics and treatment of subglottic stenosis in patients with Wegener's granulomatosis. Int J Clin Rheumatol 2010;5:475-8.

J Rheumatol 2011;38:10; doi:10.3899/jrheum.110371 